\title{
The ALMA view of the protostellar system HH212 ${ }^{\star \star \star \star}$
}

\section{The wind, the cavity, and the disk}

\author{
C. Codella ${ }^{1}$, S. Cabrit ${ }^{2,3}$, F. Gueth ${ }^{4}$, L. Podio ${ }^{1}$, S. Leurini ${ }^{5}$, R. Bachiller ${ }^{6}$, A. Gusdorf ${ }^{2}$, B. Lefloch ${ }^{3}$, B. Nisini ${ }^{7}$, \\ M. Tafalla ${ }^{6}$, and W. Yvart ${ }^{2}$
}

1 INAF, Osservatorio Astrofisico di Arcetri, Largo E. Fermi 5, 50125 Firenze, Italy e-mail: codella@arcetri.astro.it

2 LERMA, UMR 8112 du CNRS, Observatoire de Paris, École Normale Supérieure, 61 Av. de l'Observatoire, 75014 Paris, France

3 UJF-Grenoble1/CNRS-INSU, Institut de Planétologie et d'Astrophysique de Grenoble (IPAG) UMR 5274, 38041 Grenoble, France

${ }^{4}$ IRAM, 300 rue de la Piscine, 38406 Saint Martin d'Hères, France

5 Max-Planck-Institut für Radioastronomie, Auf dem Hügel 69, 53121 Bonn, Germany

${ }^{6}$ IGN, Observatorio Astronómico Nacional, Alfonso XIII 3, 28014 Madrid, Spain

7 INAF, Osservatorio Astronomico di Roma, via di Frascati 33, 00040 Monte Porzio Catone, Italy

Received 30 April 2014 / Accepted 22 July 2014

\begin{abstract}
Context. Because it is viewed simply edge-on, the $\mathrm{HH} 212$ protostellar system is an ideal laboratory for studying the interplay of infall, outflow, and rotation in the earliest stages of low-mass star formation.

Aims. We wish to exploit the unmatched combination of high angular resolution, high sensitivity, high-imaging fidelity, and spectral coverage provided by ALMA to shed light on the complex kinematics of the innermost central regions of HH212.

Methods. We mapped the inner 10" (4500 AU) of the HH212 system at $\simeq 0.5^{\prime \prime}$ resolution in several molecular tracers and in the $850 \mu \mathrm{m}$ dust continuum using the ALMA interferometer in band 7 in the extended configuration of the Early Science Cycle 0 operations.

Results. Within a single ALMA spectral set-up, we simultaneously identify all the crucial ingredients known to be involved in the star formation recipe: (i) the fast, collimated bipolar $\mathrm{SiO}$ jet driven by the protostar; (ii) the large-scale swept-up CO outflow; (iii) the flattened rotating and infalling envelope, with bipolar cavities carved by the outflow (in $\mathrm{C}^{17} \mathrm{O}(3-2)$ ); and (iv) a rotating wide-angle flow that fills the cavities and surrounds the axial jet (in $\mathrm{C}^{34} \mathrm{~S}(7-6)$ ). In addition, the compact high-velocity $\mathrm{C}^{17} \mathrm{O}$ emission $\left( \pm 1.9-3.5 \mathrm{~km} \mathrm{~s}^{-1}\right.$ from systemic) shows a velocity gradient along the equatorial plane consistent with a rotating disk of $\simeq 0$.' $2=90 \mathrm{AU}$ around $\mathrm{a} \simeq 0.3 \pm 0.1 M_{\odot}$ source. The rotating disk is possibly Keplerian.

Conclusions. HH212 is the third Class 0 protostar with possible signatures of a Keplerian disk of radius $\geq 30$ AU. The warped geometry in our CS data suggests that this large Keplerian disk might result from misaligned magnetic and rotation axes during the collapse phase. The wide-angle CS flow suggests that disk winds may be present in this source.
\end{abstract}

Key words. stars: formation - ISM: jets and outflows - ISM: molecules - ISM: individual objects: HH212

\section{Introduction}

Jets from young accreting stars remain one of the most spectacular and enigmatic phenomena in astrophysics. Although their exact launch zone is still debated, it is currently accepted that they are powered by the rotation and accretion energy of the system, and are accelerated or collimated via a magneto-hydrodynamical (MHD) process (see e.g. Ferreira et al. 2006; Shang et al. 2007; Pudritz et al. 2007, and references therein). MHD-driven jets could play a crucial role during the earliest Class 0 phase, that is, in the star and disk formation process itself. High-resolution MHD simulations of protostellar collapse with typical amounts of rotation and magnetisation show that MHD outflows are an unavoidable outcome, and are able to eject $20 \%$ to $50 \%$ of the infalling core gas before it reaches the central source (e.g. Ciardi \& Hennebelle 2010). Hence this initial phase of massive MHD

\footnotetext{
* Appendix A is available in electronic form at http: //www . aanda.org

$\star \star$ Final reduced ALMA cubes (FITS) are only available at the CDS via anonymous ftp to cdsarc.u-strasbg. fr (130.79.128.5) or via http://cdsarc.u-strasbg.fr/viz-bin/qcat?J/A+A/568/L5
}

ejection may be a key agent that can limit the final stellar mass and explain the low $(\simeq 30 \%)$ core-to-star efficiency suggested from a comparison of the initial mass function with the prestellar core mass function (e.g. André et al. 2007). However, the same simulations show that magnetic braking by the outflows and twisted $B$-fields is so efficient when the field and the spin axis $\Omega$ are aligned that Keplerian disks may be initially suppressed beyond 10 AU (e.g. Price \& Bate 2007; Machida et al. 2011). This is the so-called magnetic-braking catastrophe. On the other hand, much larger Keplerian disks of 100-150 AU have previously been reported in two Class 0 sources (L1527 and VLA1623; Tobin et al. 2010; Murillo et al. 2013). Such large Keplerian disks might result from misaligned $B-\Omega$ configurations or a strong turbulence (Joos et al. 2012, 2013), but observational evidence is still lacking. A detailed observation and characterisation of a larger sample of Class 0 infall-outflow systems is thus essential to elucidate the disk formation process, and to clarify the role of jets and outflows in removing angular momentum from the system and regulating the final stellar mass.

$\mathrm{HH} 212$ is a strikingly bright and symmetric bipolar jet from a Class 0 source in Orion (at 450 pc) that was first revealed 
in $\mathrm{H}_{2}$ imaging (Zinnecker et al. 1998). Its innermost regions have been extensively studied in $\mathrm{CO}(2-1),(3-2)$ and $\mathrm{SiO}(2-1)$, (5-4), (8-7) withf the SMA and IRAM PdBI at scales ranging from $\simeq 1^{\prime \prime}-2.5^{\prime \prime}$ (Lee et al. 2006, 2007) to $\simeq 0.3^{\prime \prime}-0.4^{\prime \prime}$ (Codella et al. 2007; Lee et al. 2008; Cabrit et al. 2007, 2012). These maps revealed a bright bipolar $\mathrm{SiO}$ microjet with inner peaks at $\pm 1-2^{\prime \prime}=450-900 \mathrm{AU}$ of the protostar, invisible in $\mathrm{H}_{2}$ due to high extinction (Codella et al. 2007; Lee et al. 2007). The $\mathrm{SiO}$ jet width $\simeq 100 \mathrm{AU}$ is remarkably close to atomic jet widths in T Tauri stars, favouring a universal MHD collimation process (Cabrit et al. 2007). Interestingly, a flattened rotating envelope in the equator perpendicular to the jet axis was observed in $\mathrm{NH}_{3}$ with a radius of $\sim 7000 \mathrm{AU}$ by Wiseman et al. (2001). Subsequent observations in $\mathrm{C}^{18} \mathrm{O}$ and ${ }^{13} \mathrm{CO}$ with the SubMillimeter Array, and in $\mathrm{HCO}^{+}$with the Atacama Large Millimeter Array (ALMA) suggest that the rotating envelope inside a radius $\sim 1000 \mathrm{AU}$ is in free-fall onto a source of mass $\simeq 0.2 M_{\odot}$ while conserving angular momentum (Lee et al. 2006, 2014). The dust continuum imaged by ALMA is flattened perpendicular to the jet and suggestive of a disk of (maximum) radius $\simeq 0.3 \mathrm{AU}$ (Lee et al. 2014). All these findings make $\mathrm{HH} 212$ an ideal laboratory for investigating the interplay of infall, outflow and rotation in the earliest stages of the low-mass starforming process.

\section{Observations}

HH212 was observed with ALMA using $2412 \mathrm{~m}$ antennas on 2012 December 1 during the Early Science Cycle 0 phase. The shortest and longest baselines were about $20 \mathrm{~m}$ and $360 \mathrm{~m}$, from which we obtained a maximum unfiltred scale of $3^{\prime \prime}$ at $850 \mu \mathrm{m}$. The $\mathrm{C}^{17} \mathrm{O}(3-2), \mathrm{SiO}(8-7)$, and $\mathrm{C}^{34} \mathrm{~S}(7-6)$ lines ${ }^{1}$ at $337061.13 \mathrm{MHz}, 347330.63 \mathrm{MHz}$, and $337396.69 \mathrm{MHz}$, respectively, were observed using spectral units of $488 \mathrm{kHz}$ $\left(0.43 \mathrm{~km} \mathrm{~s}^{-1}\right)$ resolution. Calibration was carried out following standard procedures, using quasars J0538-440, J0607-085, as well as Callisto and Ganymede. Spectral line imaging was achieved with the CASA package. Data analysis was performed using the GILDAS ${ }^{2}$ package. Images have a typical clean-beam FWHM of $0 .{ }^{\prime} 65 \times 0.0^{\prime} 47\left(\mathrm{PA}=35^{\circ}\right)$, and an rms noise of $\sim 1 \mathrm{mJy} \mathrm{beam}^{-1}$ for continuum, and $3-4 \mathrm{mJy} \mathrm{beam}^{-1}$ in the $0.44 \mathrm{~km} \mathrm{~s}^{-1}$ channels. Positions are given with respect to the MM1 protostar, located at $\alpha(\mathrm{J} 2000)=05^{\mathrm{h}} 43^{\mathrm{m}} 51^{\mathrm{s}} .41, \delta(\mathrm{J} 2000)=$ $-01^{\circ} 02^{\prime} 53^{\prime \prime} 17$, in excellent agreement with the coordinates derived by Lee et al. (2014) using ALMA.

\section{Line results and analysis}

Figure 1 compares the emission maps in the $850 \mu \mathrm{m}$ continuum, $\mathrm{SiO}(8-7), \mathrm{C}^{17} \mathrm{O}(3-2)$, and $\mathrm{C}^{34} \mathrm{~S}(7-6)$. The combination of these tracers allows us to simultaneously image in a single ALMA spectral set-up different ingredients of the star formation process: (i) a pair of narrow $\mathrm{SiO}$ jets launched from the protostar (dust peak); (ii) an extended flattened $\mathrm{C}^{17} \mathrm{O}$ envelope around the outflow waist; and (iii) biconical $\mathrm{C}^{34} \mathrm{~S}$ emission lobes surrounding the jet. The first feature is well-known characteristics of some Class 0 sources (see e.g. Tafalla et al. 2010, and references therein). A full analysis of the new details detected up by ALMA will be presented in a later publication. Here, we focus

\footnotetext{
1 Spectroscopic parameters were extracted from the Jet Propulsion Laboratory molecular database (Pickett et al. 1998) and the Cologne Database for Molecular Spectroscopy (Müller et al. 2005).

2 http://wWw.iram.fr/IRAMFR/GILDAS
}

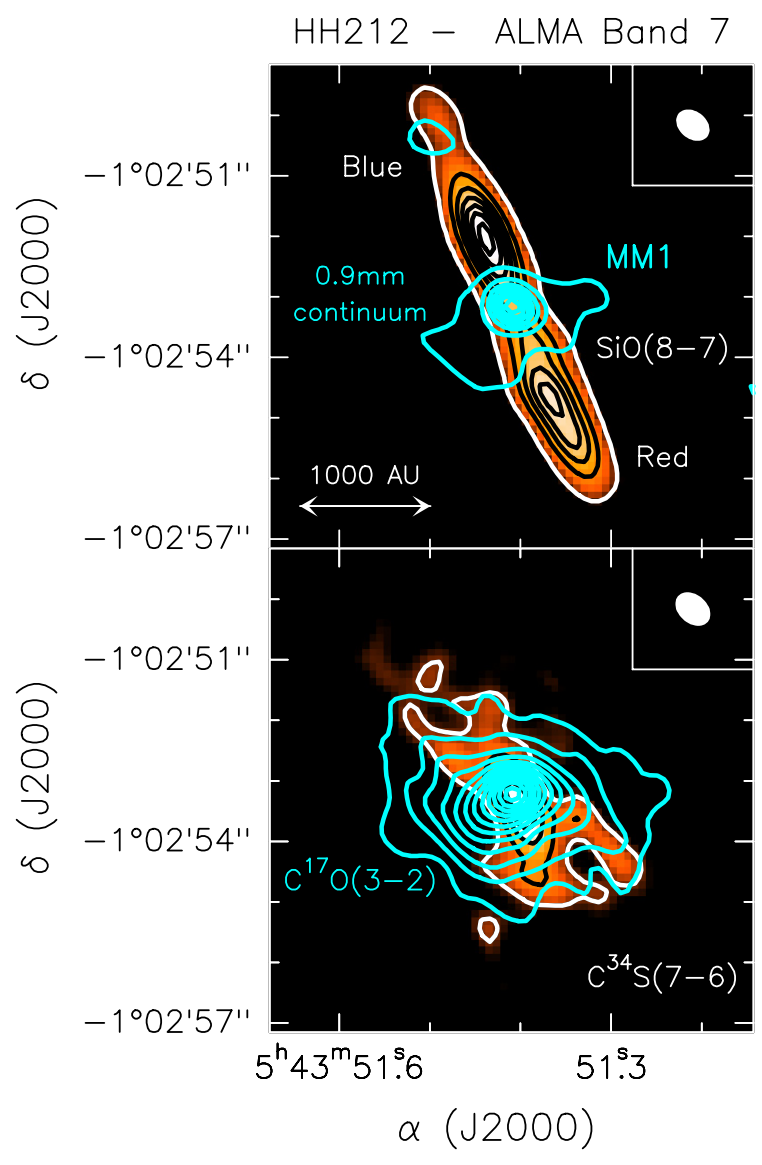

Fig. 1. Protostellar system HH212 as observed by ALMA Cycle 0 in band 7. Upper panel: $850 \mu \mathrm{m}$ continuum (turquoise contours) overlaid on $\mathrm{SiO}(8-7)$ integrated between -23 and $+15 \mathrm{~km} \mathrm{~s}^{-1}$ (colour scale and black contours), compared with the systemic velocity $V_{\text {sys }}$ (see text). First contours at $5 \sigma\left(6 \mathrm{mJybeam}^{-1}\right.$ for continuum and $270 \mathrm{mJy} \mathrm{beam}^{-1} \mathrm{~km} \mathrm{~s}^{-1}$ for $\mathrm{SiO}$ ) in steps of $20 \sigma$ (continuum) and $5 \sigma$ $(\mathrm{SiO})$. The ellipse shows the synthesised continuum HPBW $\left(0^{\prime} \cdot 61 \times\right.$ $0^{\prime \prime} 45$ at $\mathrm{PA}=51^{\circ}$ ). The beam HPBW of the SiO map is $0^{\prime} .63 \times 0.0^{\prime \prime} 46$ $\left(\mathrm{PA}=49^{\circ}\right)$. Bottom panel: contour plot of the $\mathrm{C}^{17} \mathrm{O}(3-2)$ emission (turquoise) overaid on the of the $\mathrm{C}^{34} \mathrm{~S}(7-6)$ emission (colour and black), both integrated in the $V_{\text {sys }} \pm 6 \mathrm{~km} \mathrm{~s}^{-1}$ range. The HPBW is $0{ }^{\prime} 65 \times 00^{\prime} 47\left(\mathrm{PA}=49^{\circ}\right)$. First contours and steps correspond to $5 \sigma$ (50 mJy beam ${ }^{-1} \mathrm{~km} \mathrm{~s}^{-1}$ for $\mathrm{C}^{34} \mathrm{~S}$ and $65 \mathrm{mJy}^{2}$ beam ${ }^{-1} \mathrm{~km} \mathrm{~s}^{-1}$ for $\mathrm{C}^{17} \mathrm{O}$ ).

on the latter two features, which were imaged here with unprecedented fidelity and signal-to-noise.

\subsection{Systemic velocity}

The channel maps for $\mathrm{C}^{17} \mathrm{O}(3-2)$ and $\mathrm{C}^{34} \mathrm{~S}(7-6)$ are reported in Figs. A.1 and A.2. There is no missing flux feature or P Cygni absorption near systemic velocity, in contrast to previous SMA data in the more abundant ${ }^{13} \mathrm{CO}$ and $\mathrm{C}^{18} \mathrm{O}$ molecules and ALMA data in $\mathrm{HCO}^{+}$(Lee et al. 2006, 2014). The spatial distributions clearly indicate that the velocity where emission is most spatially extended and thus presumably closest to the systemic velocity is in the $[+0.91,+1.34] \mathrm{km} \mathrm{s}^{-1}$ channel for $\mathrm{C}^{17} \mathrm{O}$, and in the $[+1.23,+1.69] \mathrm{km} \mathrm{s}^{-1}$ channel for $\mathrm{C}^{34} \mathrm{~S}$ (bottom-right panels of Figs. A.1 and A.2). These are also the channels for which the other channel maps show the best blue/red morphological symmetry. From combining these findings, and considering our spectral resolution of $\sim 0.43 \mathrm{~km} \mathrm{~s}^{-1}$, the systemic velocity $V_{\text {sys }}$, as indicated by $\mathrm{C}^{17} \mathrm{O}$ and $\mathrm{C}^{34} \mathrm{~S}$, has to be close to $\simeq+1.3 \pm 0.2 \mathrm{~km} \mathrm{~s}^{-1}$. This value is clearly supported by the 

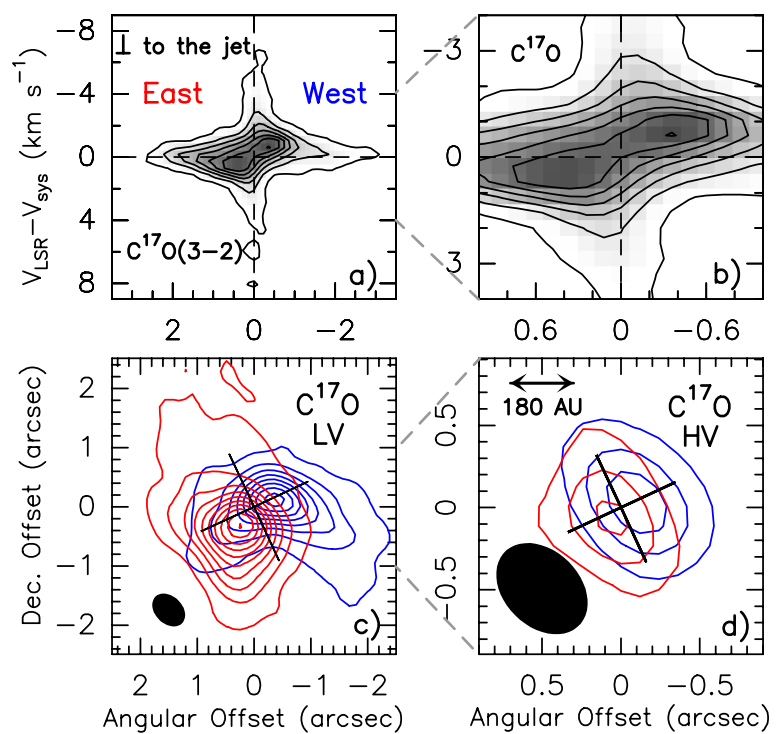

Fig. 2. Panel a): position-velocity (PV) cut of $\mathrm{C}^{17} \mathrm{O}(3-2)$ perpendicular to the jet. First contour at $5 \sigma(0.30 \mathrm{~K})$ and steps of $20 \sigma$. Dashed lines mark $V_{\text {sys }}=+1.3 \mathrm{~km} \mathrm{~s}^{-1}$ and the continuum peak MM1. Panel b): zoom-in of the $\mathrm{C}^{17} \mathrm{O}(3-2) \mathrm{PV}$ plot. Panel c): blue- and redshifted $\mathrm{C}^{17} \mathrm{O}(3-2)$ emission in the LV range $\left( \pm 0.6-1.5 \mathrm{~km} \mathrm{~s}^{-1}\right.$ from $\left.V_{\text {sys }}\right)$, tracing the rotating outflow cavity. The tilted black cross indicates the $\mathrm{SiO}$ jet direction $\left(\mathrm{PA}=22^{\circ}\right)$ and the equatorial plane. First contour at $5 \sigma$ $\left(15 \mathrm{mJy}_{\text {beam }}^{-1} \mathrm{~km} \mathrm{~s}^{-1}\right)$, then steps of $7 \sigma$. Panel $\left.\mathbf{d}\right)$ : same as panel c) for the $\mathrm{HV}$ velocity range $\left( \pm 1.9-3.5 \mathrm{~km} \mathrm{~s}^{-1}\right.$ from systemic), tracing the rotating inner disk. Note the smaller spatial scale in panels b) and d).

position-velocity (PV) diagram of the $\mathrm{C}^{17} \mathrm{O}(3-2)$ emission perpendicular to the jet axis $\left(\mathrm{PA}=112^{\circ}\right)$, see Figs. 2a,b, which shows that the emission is most extended and most symmetric about $V_{\text {sys }}=+1.3 \mathrm{~km} \mathrm{~s}^{-1}$. This value is slightly different from the $+1.6 \mathrm{~km} \mathrm{~s}^{-1}$ measured using $\mathrm{NH}_{3}(1,1)$ VLA emission by Wiseman et al. (2001). This could reflect the different spatial scales, as the ammonia core observed by Wiseman et al. (2001) has a $F W H M \sim 14000$ AU and probably traces the motion in the outer layers of the molecular envelope. We adopt $V_{\text {sys }}=+1.3 \mathrm{~km} \mathrm{~s}^{-1}$ in the remainder of this paper.

\section{2. $C^{17} \mathrm{O}(3-2)$ : rotating envelope cavities and inner disk}

Figure $2 \mathrm{ab}$ shows that, in addition to infall motions (traced by the extended diamond shape of the PV at low contour levels), a rotation signature is clearly seen in the form of two emission peaks at low velocities $\leq 1.5 \mathrm{~km} \mathrm{~s}^{-1}$ (referred to as LV in the following): one blueshifted to the west, and one redshifted to the east. Figure $2 \mathrm{c}$ shows that this rotating $\mathrm{LV} \mathrm{C}^{17} \mathrm{O}$ emission is (mainly) tracing the sides of the southern cavity carved by the outflow into the envelope. The sides are rotating in the same sense as the $\mathrm{NH}_{3}, \mathrm{C}^{18} \mathrm{O}$, and $\mathrm{HCO}^{+}$cores (Wiseman et al. 2001; Lee et al. 2006, 2014), that is with blueshifted gas in the west and redshifted gas in the east, as expected for envelope material swept-up and compressed by the outflow.

Figure $2 \mathrm{~d}$ shows that at higher velocities $\simeq 1.9-3.5 \mathrm{~km} \mathrm{~s}^{-1}$ from systemic (denoted as HV in the following): (i) the E-W velocity gradient is still present; and (ii) the emission is definitely more compact $(\leq 0,5)$. Similarly to other Class 0 disk studies (see e.g. Murillo et al. 2013, and references therein), the emission centroid positions in each channel were obtained from

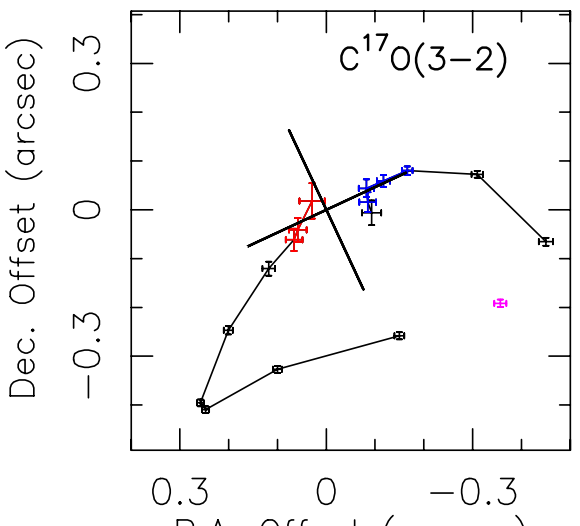

R.A. Offset (arcsec)

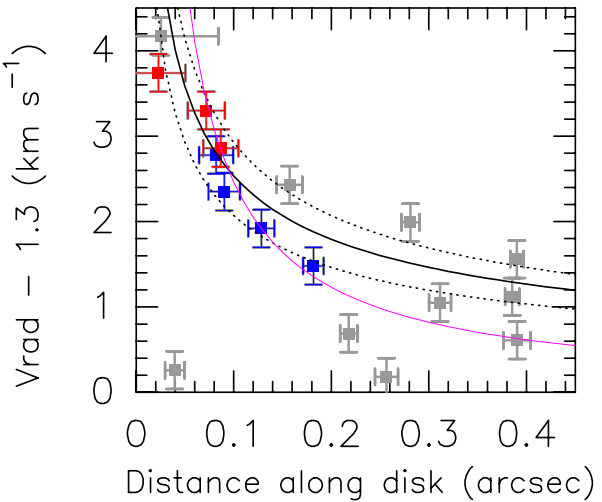

Fig. 3. Upper panel: distribution of the $\mathrm{C}^{17} \mathrm{O}(3-2)$ centroid positions (from fits in the $u v$ domain with $1 \sigma$ error bars) in the various velocity channels (Fig. A.1). Red and blue datapoints denote the channels whose fitted centroids lie, within the error bars, on the equator. Bottom panel: velocity shift from $V_{\text {sys }}$ as a function of distance from the protostar along the equator $\left(\mathrm{PA}=122^{\circ}\right)$. Red and blue datapoints denote the channels whose fitted centroids lie along the equator (see upper panel). The assumed systemic velocity is $+1.3 \mathrm{~km} \mathrm{~s}^{-1}$ (see text). Grey points refer to $\mathrm{LV}$ emission tracing the $\mathrm{C}^{17} \mathrm{O}$ envelope and cavity. Black solid and dotted curves show Keplerian rotation around a stellar mass of $0.3 \pm 0.1 M_{\odot}$. The $r^{-1}$ curve for angular momentum conservation is plotted in magenta for comparison.

elliptical Gaussian fits in the $u v$ domain $^{3}$. The results are plotted in Fig. 3. While the centroids in the LV range fall on the southern cavity, as expected from the channel maps, the centroids in the $\mathrm{HV}$ range move to the equatorial plane (within the uncertainties), which indicates an inner rotating equatorial disk of radius $\simeq 0$ '. 2 (90 AU). These findings are consistent with the outer radius of the flattened continuum disk imaged by Lee et al. (2014), who derived $120 \mathrm{AU}$ using their ALMA map at $350 \mathrm{GHz}$.

We compare in Fig. 3 the centroid measurements along the disk plane with Keplerian rotation curves and, as a reference, with $a \sim r^{-1}$ rotation curve that conserves specific angular momentum (pseudo-disk). Although $a \sim r^{-1}$ trend is not ruled out, Fig. 3 shows that the present measurements are consistent with Keplerian rotation out to $\simeq 0 . ' 2=90 \mathrm{AU}$ around a $0.3 \pm 0.1 M_{\odot}$ protostar. This mass estimate agrees with the $0.2 M_{\odot}$ derived by Lee et al. $(2006,2014)$ from modelling the rotating infall kinematics of $\mathrm{C}^{13} \mathrm{O}, \mathrm{C}^{18} \mathrm{O}$, and $\mathrm{HCO}^{+}$on larger scales ${ }^{4}$ All these

3 We used the GILDAS $u v$ - fit task: the resulting error on centroid position is the function of the channel signal-to-noise ratio and atmospheric seeing, and is typically much smaller than the beam size.

4 The assumption of free-fall (used by Lee et al. 2014) overestimates the true infall speed in the presence of rotation (see e.g. Stahler et al. 1994; Sakai et al. 2014), and thus tends to underestimate the required central mass. 


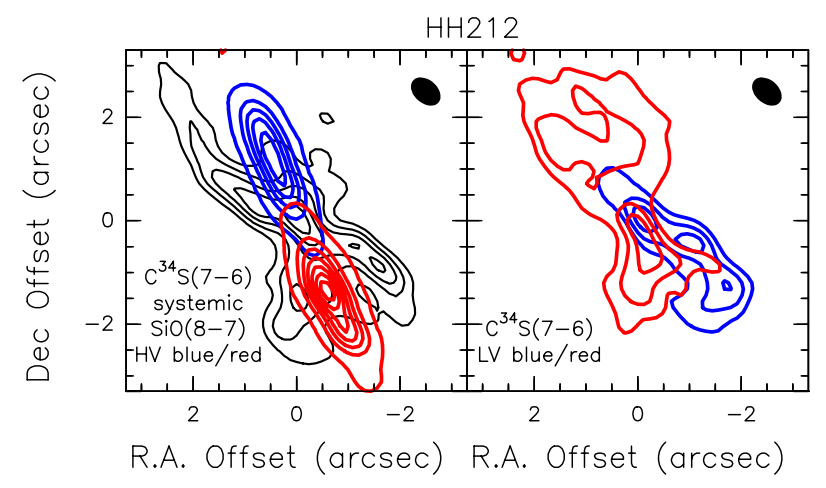

Fig. 4. Left: $\mathrm{SiO}(8-7)$ channel maps at $V_{\text {sys }} \pm 8 \mathrm{~km} \mathrm{~s}^{-1}$ (blue and red) overlaid on top of $\mathrm{C}^{34} \mathrm{~S}(7-6)$ close to systemic velocity (black). Right: $\mathrm{C}^{34} \mathrm{~S}(7-6)$ channel maps at low blue and redshifted velocities $\sim V_{\text {sys }} \pm 0.9 \mathrm{~km} \mathrm{~s}^{-1}$, showing a rotating wide-angle flow about the jet axis. $\mathrm{SiO}$ first contour at $5 \sigma\left(25 \mathrm{mJy} \mathrm{beam}^{-1} \mathrm{~km} \mathrm{~s}^{-1}\right)$ and steps of $25 \sigma$. $\mathrm{C}^{34} \mathrm{~S}$ first contours and steps correspond to $5 \sigma\left(15 \mathrm{mJy} \mathrm{beam}^{-1} \mathrm{~km} \mathrm{~s}^{-1}\right)$.

findings support the hypothesis of a rotating disk of radius $90 \mathrm{AU}$ around the MM1 protostar, possibly in Keplerian rotation inside this radius.

\section{3. $C^{34} S$ : warped cavity and wide-angle flow}

While the bulk of the $\mathrm{C}^{17} \mathrm{O}$ emission in Fig. 1 (Middle) is tracing the protostellar envelope of $F W H M \simeq 460$ AU flattened in the equatorial plane, $\mathrm{C}^{34} \mathrm{~S}$ is elongated along the outflow, with little emission in the equatorial plane. Given the high critical density of the $\mathrm{C}^{34} \mathrm{~S}(7-6)$ line $\left(\sim 9 \times 10^{6} \mathrm{~cm}^{-3}\right.$ between $10 \mathrm{~K}$ and $300 \mathrm{~K}$, according to the collisional rates of Lique et al. 2006), and the envelope density inferred by Lee et al (2014), this suggests that $\mathrm{CS}$ is tracing a dense gas component more closely related to the primary jet or outflow. Near systemic velocity, $C^{34} S$ is imaging a biconical structure surrounding the $\mathrm{SiO}$ jet and with MM1 at the vertex (see Fig. 4). A surprising finding is the S-shaped warp seen in $\mathrm{C}^{34} \mathrm{~S}$, despite the very straight axial jet. Similar cavity asymmetries are predicted by MHD simulations during protostellar collapse with a misaligned magnetic field and angular momentum vectors (e.g. Ciardi \& Hennebelle 2010); hence the $C^{34} \mathrm{~S}$ warp might be a remnant imprint of this initial configuration, which has been invoked to explain the formation of large Keplerian disks in Class 0 sources (Joos et al. 2012). Sensitive polarisation measurements with ALMA will be crucial to test this hypothesis.

Figure 4 further indicates that the $\mathrm{C}^{34} \mathrm{~S}$ southern lobe is clearly rotating about the jet in the same sense as the $\mathrm{C}^{17} \mathrm{O}$ cavity. However, Fig. A.2 shows that the structure of $\mathrm{C}^{34} \mathrm{~S}$ is narrower than that of $\mathrm{C}^{17} \mathrm{O}$ and becomes gradually more collimated towards the jet axis as the velocity increases, changing progressively from a biconical morphology near $V_{\text {sys }}$ to a jet at $V_{\text {LSR }}-V_{\text {sys }} \geq 2.5 \mathrm{~km} \mathrm{~s}^{-1}$. This suggests that the $\mathrm{C}^{34} \mathrm{~S}$ emission may be filling-in the swept-up extended cavity delineated by $\mathrm{C}^{17} \mathrm{O}$, and trace a rotating wide-angle flow with a nested onion-like velocity structure, highly reminiscent of that seen in the atomic jet from the T Tauri star DG Tau (Bacciotti et al. 2002).

\section{Conclusions}

$\mathrm{C}^{17} \mathrm{O}$ traces three different components that depend on the velocity of its emission: the infalling envelope near systemic velocity, the rotating cavity in the LV range, and a rotating equatorial disk in the HV range with radius $\sim 0{ }^{\prime} 2=90 \mathrm{AU}$, which may be Keplerian around a protostar of $0.3 \pm 0.1 M_{\odot}$. On the other hand, the wide-angle CS flow suggests that disk winds may be present in this source. The present results calls for more observations at high spatial and spectral resolutions of disk tracers to confirm the disk size and verify its Keplerian nature.

Acknowledgements. We are grateful to D. Galli for helpful discussions and suggestions. This paper makes use of the following ALMA data: ADS/JAO.ALMA\#2011.0.000647.S (PI: C. Codella). ALMA is a partnership of ESO (representing its member states), NSF (USA) and NINS (Japan), together with NRC (Canada) and NSC and ASIAA (Taiwan), in cooperation with the Republic of Chile. The Joint ALMA Observatory is operated by ESO, AUI/NRAO and NAOJ. This work was partly supported by the PRIN INAF 2012 - JEDI and by the Italian Ministero dell'Istruzione, Università e Ricerca through the grant Progetti Premiali 2012 - iALMA. L.P. has received funding from the European Union Seventh Framework Programme (FP7/2007-2013) under grant agreement No. 267251.

\section{References}

André, Ph., Belloche, A., Motte, F., \& Peretto, N. 2007, A\&A, 472, 519 Bacciotti, F., Ray, T. P., Mundt, R., Eislöffel, J., \& Solf, J. 2002 ApJ, 576, 222 Cabrit, S., Ferreira, J., \& Raga, A. C. 1999, A\&A, 343, L61

Cabrit, S., Codella, C., Gueth, F., et al. 2007, A\&A, 468, L29

Cabrit, S., Codella, C., Gueth, F., \& Gusdorf, A. 2012, A\&A, 548, L2

Ciardi, A., \& Hennebelle, P. 2010, A\&A, 409, L39

Claussen, M. J., Marvel, K. B., Wootten, A., \& Wilking, B. A. 1998, ApJ, 507, L79

Codella, C., Cabrit, S., Gueth, F., et al. 2007, A\&A, 462, L53

Ferreira, J., Dougados, C., \& Cabrit, S. 2006, A\&A, 453, 785

Joos, M., Hennebelle, P., \& Ciardi, A. 2012, A\&A, 543, A128

Joos, M., Hennebelle, P., Ciardi, A., \& Fromang, S. 2013, A\&A, 554, A17

Lee, C.-F., Ho, P. T. P., Beuther, H., et al. 2006, ApJ, 639, L292

Lee, C.-F., Ho, P. T. P., Hirano, N., et al. 2007, ApJ, 659, L499

Lee, C.-F., Ho, P. T. P., Bourke, T. L., et al. 2008, ApJ, 685, 1026

Lee, C.-F., Hirano, N., Zhang, Q., et al. 2014, ApJ, 786, 114

Lique, F., Spielfiedel, A., \& Cernicharo, J. 2006, A\&A, 451, 1125

Machida, M. N., Inutsuka, S.-I., \& Matsumoto, T. 2011, PASJ, 63, 555

Müller, H. S. P., Schöier, F. L., Stutzki, J., \& Winnewisser, G. 2005, J. Mol. Struct., 742, 215

Murillo, N. M., Lai, S.-P., Bruderer, S., Harsolo, D., \& van Dishoeck, E. F. 2013, A\&A, 560, A103

Pickett, H. M., Poynter, R. L., Cohen, E. A., et al. 1998, J. Quant. Spectr. Radiat. Transf., 60, 883

Price, D. J., \& Bate, M. 2007, Ap\&SS, 311, 75

Pudritz, R. E., Ouyed, R., Fendt Ch., \& Brandenburg, A. 2007, in Protostars and Planets V (Tucson: University of Arizona Press ), 277

Sakai, N., Sakai, T., Hirota, T., et al. 2014, Nature, 507, 78

Shang, H., Li, Z.-Y., \& Hirano, N. 2007, in Protostars and Planets V (Tucson: University of Arizona Press), 261

Stahler, S., Korycansky, D. G., Brothers, M. J., et al. 1994, ApJ, 431, 341

Tafalla, M., Santiago-García, J., Hacar, A., \& Bachiller, R. 2010, A\&A, 521, A91

Tobin, J., Hartmann, L., Looney, L. W., \& Chiang, H.-F. 2010, ApJ, 712, 1010

Wiseman, J., Wootten, A., Zinnecker, H., \& McCaoughrean, M. 2001, ApJ, 550, L87

Zinnecker, H., McCaughrean, M. J., \& Rayner, J. T. 1998, Nature, 394, 862

Page 5 is available in the electronic edition of the journal at http://www . aanda.org 


\section{Appendix $\mathrm{A}: \mathrm{C}^{17} \mathrm{O}, \mathrm{C}^{34} \mathrm{~S}$, and $\mathrm{CH}_{3} \mathrm{OH}$ kinematics}

We report in Figs. A.1 and A.2 the channel maps of the $\mathrm{C}^{17} \mathrm{O}(3-2)$ and $\mathrm{C}^{34} \mathrm{~S}(7-6)$ (continuum-subtracted) emissions towards HH212 that were analysed and discussed in the main text.

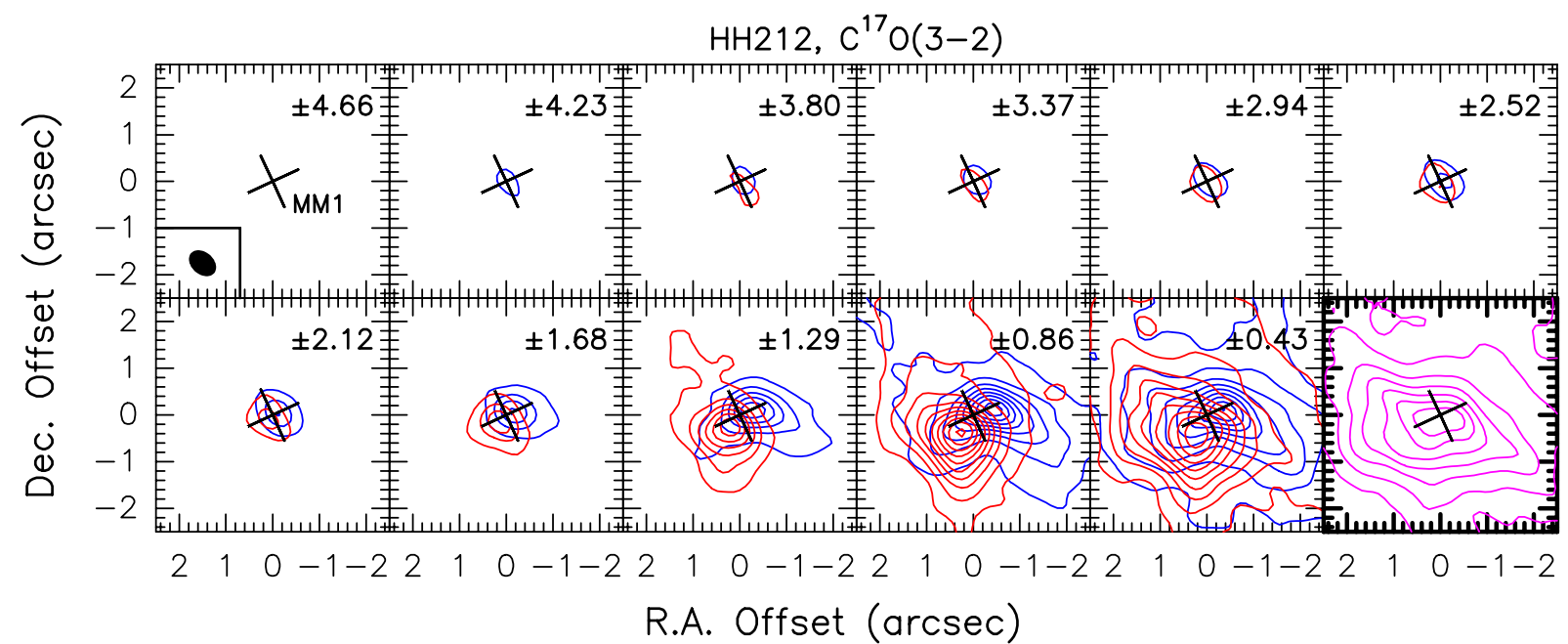

Fig. A.1. Channel maps of the $\mathrm{C}^{17} \mathrm{O}(3-2)$ (continuum-subtracted) emissions towards $\mathrm{HH} 212$. The bottom-right panel shows in magenta the $0.43 \mathrm{~km} \mathrm{~s}^{-1}$ wide channel (centred on $+1.13 \mathrm{~km} \mathrm{~s}^{-1}$ ) where emission is most spatially extended. The other panels superposed in blue and red contours are the channel maps at symmetric blue/red velocity offsets from this central channel, with the velocity shift given (in $\mathrm{km} \mathrm{s}^{-1}$ ) in the upperright corner. The black cross (inclined to show the SiO jet direction) indicates the position of the MM1 continuum source. The ellipse in the top-left panel shows the ALMA synthesised beam (HPBW): $0{ }^{\prime} 65 \times 00^{\prime} 47\left(\mathrm{PA}=49^{\circ}\right)$. First contours and steps correspond to $5 \sigma\left(15 \mathrm{mJy} \mathrm{beam}^{-1} \mathrm{~km} \mathrm{~s}^{-1}\right)$.

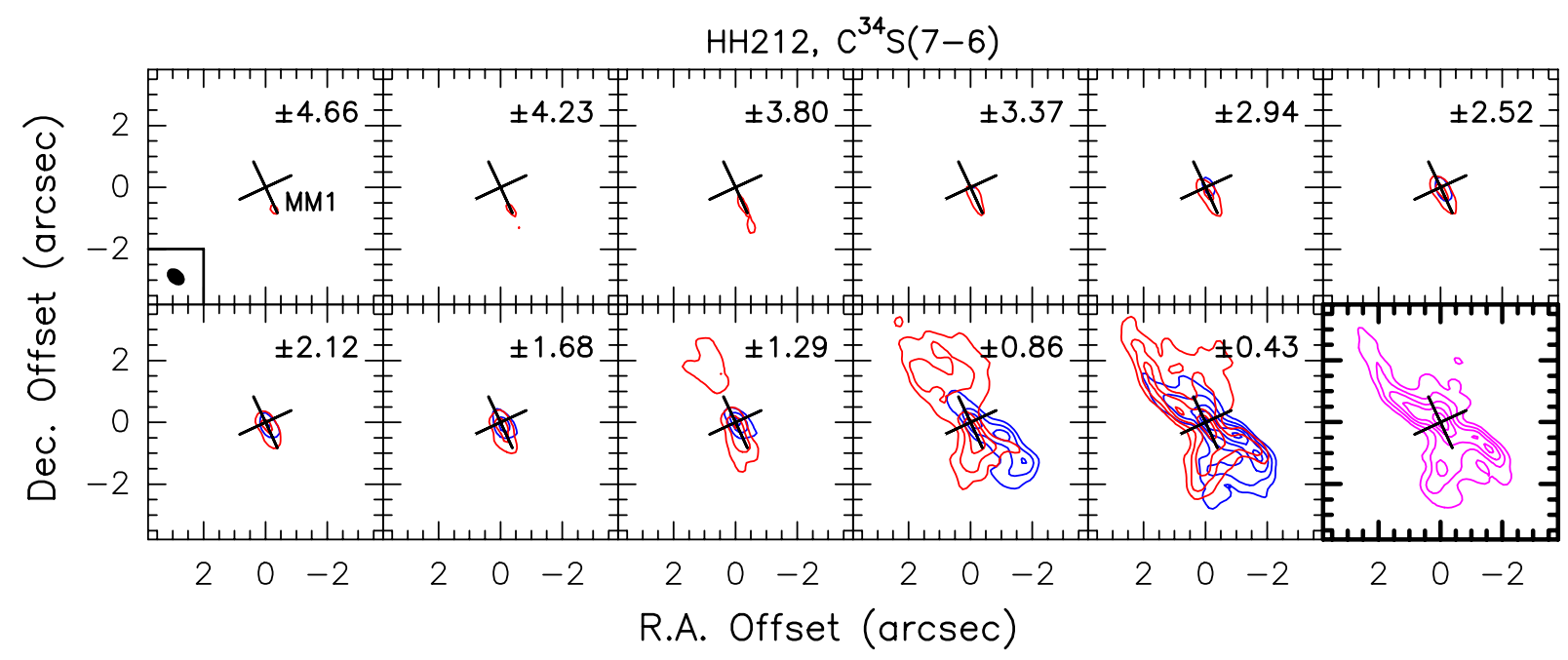

Fig. A.2. Channel maps of the $\mathrm{C}^{34} \mathrm{~S}(7-6)$ blue- and redshifted (continuum-subtracted) emissions towards HH212. The bottom-right panel shows in magenta the $0.43 \mathrm{~km} \mathrm{~s}^{-1}$ wide channel (centred on $+1.42 \mathrm{~km} \mathrm{~s}^{-1}$ ) where emission is the most spatially extended. The other panels superposed in blue and red contours are the channel maps at symmetric blue and red velocity offsets from this central channel, with the velocity shift given (in $\mathrm{km} \mathrm{s}^{-1}$ ) in the upper-right corner. Symbols are the same as in Fig. A.1. First contours and steps correspond to $5 \sigma\left(15 \mathrm{mJy} \mathrm{beam}^{-1} \mathrm{~km} \mathrm{~s}^{-1}\right)$. 